\title{
El «indígena autorizado" y el «indígena desautorizado»: categorías paradójicas producidas colateralmente en el discurso de los derechos culturales en Ecuador ${ }^{1}$
}

\author{
The "authorized indigenous» and the \\ «unauthorized indigenous»: paradoxical categories collaterally \\ produced in Ecuadorian cultural rights discourse
}

Stephanie León Calle

Universidad Pablo de Olavide

tefyanturio@hotmail.com

Ana Karen Poveda-Bustillos

Universidad Internacional del Ecuador

karenpoveda65@gmail.com

Sumario: Introducción. 1. Revisión sumaria de la categorización e identificación de la población indígena durante la colonia. 1.1. Primera etapa. 1.2. Segunda etapa. 1.2.1. Caso del Comendador Perafán de Rivera vs. Pedro. 2. Revisión sumaria de la normativa ecuatoriana relativa a los pueblos indígenas. 2.1. El primer censo ecuatoriano (1950). Conclusiones. Bibliografía.

Resumen: Históricamente la asignación de la categoría «indio» ha sido construida desde el poder, con expertos del Estado y gobierno encargados de identificarlos. Actualmente el criterio para el reconocimiento de los derechos de los pueblos indígenas es la diferencia cultural, tanto la legislación internacional como la ecuatoriana establecen que el método para la identificación es la autodefinición. Al discurso de los derechos de la diferencia le es inherente un modelo de alteridad oficial que ha sido construido en base a estereotipos (el indígena debe vestir, hablar y danzar tradicionalmente). Se vislumbran las dificultades que acompañan al criterio de autodefinición en el Ecuador y se evidencia que sin importar cuál sea el criterio, para efectos de la identificación se encuentran supeditados al esencialismo (racial y cultural),

1 Agradecemos la entrevista realizada a la historiadora Laura Giraudo y la colaboración de Patricia Ordoñez León cuyos aportes y reflexiones históricas fueron imprescindibles para abrir y el desarrollo de este análisis. 
así como a reproducir el modelo oficial del ser indígena, dando lugar a dicotomías estereotipadas del discurso hegemónico que podríamos acuñar como el «indígena autorizado» y el «indígena desautorizado» (el que no es suficientemente indígena para merecer estos derechos).

Palabras clave: esencialismo cultural, políticas culturales, autoidentificación, pueblo indígena, derechos culturales.

Abstract: Throughout history, the status of «indian» as a category was constructed from a position of power, with colonial burocracy experts that were in charge of establishing who was or was not considered «indian». Currently, the criteria for the recognition of indigenous people's rights is cultural difference. Both, the international and the Ecuadorian legislation, establish that the method for the identification is self-definition. These rights that allow to differentiate are accompanied by a model of official alterity that has been based on the construction of stereotypes (the indigenous must dress, speak and dance traditionally). The difficulties that accompany the criteria of self-definition in Ecuador are glimpsed, showing that no matter which method is used to define and to identify, they are subject to the essentialization of culture and to reproducing the official model of the indigenous being. As a consequence, this gives place to stereotyped dichotomies of the hegemonic discourse that could be referred as who's to be the "authorized indigenous» and or who's to be the "unauthorized indigenous».

Keywords: cultural essentialism, cultural policies, self-identification, indigenous people, cultural rights, patrimonialistic culture. 


\section{Introducción}

A lo largo de la historia, durante el proyecto colonial, tanto en la fundación de los Estados latinoamericanos como en el desarrollo del proyecto nacional en el Ecuador, se ha desplegado un modelo homogenizante y se ha amparado únicamente al grupo hegemónico y su cultura. En el proyecto de nación ecuatoriana explícita e implícitamente se ha anhelado y planteado la desaparición de la cultura indígena, acometiendo políticas que han ido desde el repoblamiento, desposeimiento del territorio, hasta la imposición cultural. Ante la necesidad de contar con derechos especiales para la protección de la diferencia cultural han surgido derechos específicos para proteger la diferencia, ya que si las personas pudieran desarrollar libremente su cultura, ni siquiera se habría suscitado el debate de la pertinencia de estos derechos, menos aún su proclamación. La premisa de estos derechos se encuentra establecida en las teorías de la igualdad y equidad: un grupo culturalmente diferenciado requiere un tratamiento político y jurídico diferenciado. Sin embargo, colateralmente a la legislación relativa a los derechos de la diferencia cultural se produce una serie de complejidades derivadas del criterio fundacional de estos derechos, que es la autodefinición.

La autodefinición o la conciencia de identidad se encuentra asociada a las tradiciones y costumbres diferentes. Es decir, el criterio o perspectiva de la diferenciación es cultural y ya no racial. Sin embargo, a lo largo de este documento se analizará que en el criterio de la autoidentificación todavía palpita el imaginario colonial sobre los «indios», pues se espera que cumplan estereotipos o el modelo de alteridad oficial e ideal que se ha establecido en Ecuador. Por ello, colateralmente surge la necesidad de líderes y colectivos indígenas de inscribir la autodefinición según el mandato de la percepción dominante, pues se los aboca a reconocerse a partir de significados dominantes basados en discursos sobre la identidad, generándose la necesidad forzosa de "conservarse» como estrategia de legitimación. En este sentido, al discurso de los derechos culturales, le acompaña inherentemente una perspectiva esencialista que permite la construcción de un modelo de alteridad oficial e ideal, que a su vez sostiene la diferenciación de quienes «merecen» los derechos culturales (quienes son los suficientemente indígenas) y quienes no están en condiciones de gozarlos a cabalidad.

Una vez reseñados los problemas colaterales al discurso de los derechos culturales, que serán analizados posteriormente, referenciemos 
brevemente información histórica, ya que la categorización de la población indígena responde a una historia de antigua data que inició en la época colonial y que se encuentra plasmada en distintos soportes: escritos literarios, crónicas, correspondencia epistolar, entre otros. Los criterios de identificación han sido construidos desde la visión hegemónica, que se atribuía la competencia de asignarla y ha sido supeditada a la subjetividad de quienes los identificaban según sus percepciones e interpretaciones, creando distintas categorizaciones a lo largo de la historia: indios, aborígenes, pueblos tribales. En el periodo colonial se dudó de la naturaleza humana de los indígenas y cuando se determinó su humanidad, decretándose su libertad, en el derecho indiano se plasmó que jurídicamente eran incapaces relativos; esta concepción persistió hasta mediados del siglo xx en el marco del derecho internacional, en el cual se hablaba de pueblos "semitribales», es decir colectivos atrapados en una etapa de civilización poco avanzada.

Actualmente en la legislación contemporánea internacional y en el Ecuador se utiliza la categoría «pueblos indígenas» y a partir de mediados del siglo xx desde la expedición del Convenio 169 de la OIT se ha dejado atrás el criterio de identificación externa, abriendo paso al criterio de «autoidentificación», es decir, la identificación basada en el reconocimiento fundamentado en la cultura, con lo cual aparentemente se zanja la tradicional manera de definirlos racial y genealógicamente por terceros, dando paso a la concepción cultural de la identidad.

Después de las consecuencias nefastas derivadas de la II Guerra Mundial, una vez desacreditado el concepto biológico de la raza, éste fue eliminado en la ONU. Desaparecido este concepto, surgió la cultura como un nuevo criterio de diferenciación entre grupos humanos. Por ello la legislación contemporánea relativa a derechos indígenas tiene su base en la diferenciación cultural. Precisemos que la legislación internacional de Derechos Humanos referida es el Convenio Nro. 169 de la OIT $^{2}$ sobre Pueblos Indígenas y Tribales (1991) y la Declaración de las Naciones Unidas sobre los Derechos de los Pueblos Indígenas (2007), los dos instrumentos se basan en el criterio de autodefinición, reconocen el derecho de todos los

2 En este convenio se introdujo la categoría «pueblos», sustituyendo la categoría de «poblaciones tribales» establecido en el Convenio No. 107 que anteriormente regulaba la misma materia —el mismo quedó obsoleto por su visión colonial e integracionista que consideraba al indígena atrapado en una etapa «menos avanzada»—. 
pueblos a ser diferentes, a considerarse a sí mismos diferentes y a ser respetados como tales (DNUDPI, Art. 1) . $^{3}$

Rodolfo Stavenhagen señala que la autodefinición es una herramienta política de los pueblos indígenas para obtener un estatus o categoría que no sea construido unilateralmente por los gobiernos, a saber:

Ahora se ha transformado en una cuestión de identidad cultural, y con frecuencia un asunto de honor (independientemente de criterios tan «objetivos» como el uso de la lengua, el vestido, o la participación activa en la vida de la comunidad). Más que una elección individual, se trata para muchas organizaciones del reconocimiento grupal y de la identidad colectiva (Stavenhagen 2010, 60).

El criterio fundacional de los derechos de los pueblos indígenas es la autodefinición, cuya base se encuentra en la conciencia de identidad, que a su vez se basa en la diferencia cultural. Es decir, la conciencia de identidad se sustenta en criterios culturales: étnicos, lingüísticos, costumbres, derecho consuetudinario, vida en comunidad, medicina tradicional. Consecuentemente, se traslapan el criterio de autoidentificación y la identidad indígena asociada con varios referentes culturales (vivienda, vestimenta, medicina tradicional, territorio, entre otros).

3 En el Informe del Relator Especial de las Naciones Unidas, José Martínez Cobo, titulado: «Estudio del problema de la discriminación contra las poblaciones indígenas», se caracteriza a los pueblos indígenas señalando: son comunidades, pueblos y naciones indígenas los que, teniendo una continuidad histórica con las sociedades anteriores a la invasión y precoloniales que se desarrollaron en sus territorios, se consideran distintos a otros sectores de las sociedades que ahora prevalecen en esos territorios o en parte de ellos. Constituyen ahora sectores no dominantes de la sociedad y tienen la determinación de preservar, desarrollar y transmitir a futuras generaciones sus territorios ancestrales y su identidad étnica como base de su existencia continuada como pueblo, de acuerdo con sus propios patrones culturales, sus instituciones sociales y sus sistemas legales (ONU, doc. núm. E/CN.4/Sub.2/1986/87) Se entiende entonces que esta caracterización, además de lo mencionado sobre la continuidad histórica, hace referencia a otros elementos como la singularidad, al hecho de que no pueden (comunidades, pueblos y naciones indígenas) tener un carácter dominante en sociedad y la determinación de pasar a las generaciones venideras su territorio e identidad étnica El foro permanente de las Naciones Unidas para Cuestiones Indígenas ha añadido tres puntos que se pueden sumar a los contenidos en la caracterización del informe Cobo: un vínculo fuerte con el territorio y los recursos naturales circundantes y una singularidad en dos aspectos: en primer lugar, en cuanto a sus sistemas sociales, económicos y políticos; y, en segundo lugar, en cuanto idiomas, cultura y creencias singulares. 
Si bien los derechos basados en la diferencia de los pueblos son necesarios frente a las políticas de asimilación del estado (blancomestizo), en el discurso jurídico internacional y en el Ecuador, los pueblos excluidos en la conformación del Estado nación ahora se encuentran llamados a cumplir la obligatoriedad de ser visiblemente diferentes y generalmente cumplir los estereotipos raciales con los cuales suelen ser representados para poder reivindicar sus derechos. El criterio de la diferenciación se establece en razón del contraste cultural, lo cual genera una presión de ser y parecer distinguibles y de cumplir ciertos atributos o «muestras legitimadoras de identidad indígena», ya que se espera que el indígena hable, vista, viva, dance de manera generalmente estereotipada para que pueda ser legítimo titular de sus derechos colectivos.

Este discurso e imaginario dominantes que hacen del ser indígena una especie de patrimonio cultural inmutable, en parte son un resultado del imaginario colonial, como un producto construido tanto por el poder gubernamental como por la academia, las ciencias sociales (específicamente por la tradición antropológica y el neoidigenismo de los años 70).

Parafraseando y trasplantando para nuestro tema la perspectiva de Said sobre el orientalismo, podría decirse que el indígena muchas veces resulta ser el resultado del discurso occidental y de la estrategia de la «otrerización», construimos mediante estereotipos la alteridad esencial constitutiva del indígena. Esta estrategia no se limita a establecer una diferencia esencial, irreductible e insalvable, sino que también establece una jerarquía que pone a la sociedad dominante en la cúspide (Said, citado por Restrepo 2004, 81), renovándose una suerte de jerarquías culturales que posibilitan la continuidad de la dominación.

La asociación de la identidad indígena al cumplimiento de generalidades estereotipadas de lo que se cree que es ser indígena suscita una suerte de presión a las comunidades, para asegurar sus reivindicaciones necesitan exotizarse y regular la tradición de manera cosificante generando que se dictaminen normas culturales cosificantes. Por ello, los representantes de colectivos indígenas a menudo suelen presentar sus demandas en el marco cultural, defendiendo las prácticas tradicionales, posicionándose por su diferencia cultural, al registrar autodefinición se ven presionados a regular la tradición de manera coercitiva (penalizándose por ejemplo el abandono de la vestimenta tradicional a las mujeres). El establecimiento de pautas étnicas inmutables se torna una estrategia, de lo contrario podría suscitarse una crisis de legitimidad de sus derechos frente a la cultura dominante. 
En algunos documentos comunitarios en los que se intenta regular la tradición se exige la conservación de características externas de la identidad, por ejemplo, en el estatuto de 1975 de la nacionalidad Tsáchila ${ }^{4}$ se les conmina a conservar las características más externas de su identidad como la costumbre de pintarse el cabello de rojo para los hombres o de vestirse con su atuendo típico las mujeres; en la reforma de 1983 se regula la prohibición de contraer matrimonios interétnicos (Ventura 2012, 100)

Es común que en el imaginario social y burocrático perviva la idea racial de que una persona, para ser considerada indígena, deba ser descendiente de «indígenas puros» y habitar en un territorio ancestral, ${ }^{6}$ además, suele considerarse la obligatoriedad de la conservación de la propiedad comunal ya que generalmente se ha promovido la idea de que para ser indígena se debe vivir únicamente en régimen de propiedad colectiva, negando así a una parte de esta población que ha migrado o se ha refugiado en zonas urbanas marginales.

La categoría indígena también es una construcción social7, un producto conceptual, una forma genérica que no describe una pertenencia étnica concreta. Pese a ello, suele ser utilizada como si describiera una realidad homogénea (en Ecuador suele asociarse lo "andino» como sinónimo de lo «indígena»). La concepción de la cultura en el derecho supone la existencia de grupos identificables por elementos esenciales. Generalmente las reglas que pretenden traducir jurídicamente la identidad indígena revelan una perspectiva esencialista de la cultura (Grammond 2014, 310, 313, 338).

4 Conocido por el término castellano de los Colorados por su costumbre de pintarse el cuerpo y el cabello de color rojo con achiote, quienes habitan en el cantón Santo Domingo de los Colorados (Ventura 1997, 1).

5 Para ampliar esta referencia se puede leer la investigación realizada por Monserrath Ventura $(2012,101)$.

6 Estos fundamentos raciales de la utilización de criterios genealógicos también se encuentran presentes en varios discursos de pueblos indígenas, lo cual da cuenta de que este criterio que regía el estatus de indígena en la colonia ha sido interiorizado. Sin embargo, considerando que la familia es el principal vector de transmisión de la cultura sí sería posible definir la identidad por geneaología sin caer en racismo (Juteau citado en Grammond 2014, 317).

7 Amerita citar la declaración de un joven dirigente runa de Cotopaxi, que da cuenta de que el concepto actual de «indígena» es un producto óptico e ideológico, debido a la incompresión del «otro»: «Nosotros no somos ni indígenas, ni nativos, ni aborígenes, ni indios... Indios son los de la india. Esto es lo que nos dicen que somos, pero nosotros nos decimos quichuas, shuar, achuar... Si nosotros nos llamamos indígenas hoy, es porque nos hemos apropiado lo que los demás nos llaman, para llamarnos así nosotros mismos. Pero esto ya es político; la afirmación de nuestras diferentes nacionalidades frente al Estado y sociedades nacionales (A.I. en Sánchez 2013, 111) 
La autodefinición o descripción de las características culturales en documentos oficiales para obtener determinados derechos (para realizar, por ejemplo, la inscripción oficial de un colectivo indígena) es una labor compleja, ya que de la vida cotidiana a los papeles puede existir una distancia que demanda ser ajustada para lograr su compatibilidad. La necesidad de afirmar demandas en formas compatibles con el discurso con intensos referentes étnicos conlleva una tendencia hacia la esencialización y la idealización de las prácticas y realidades indígenas (Giraudo 2007, 38), decretándose medidas rígidas que incluso en ocasiones provocan pérdida de interés de vivir en comunidad, amenazando a la cohesión y reproducción de los pueblos. Podríamos afirmar que la libertad de "autodefinición» se vicia al ser abocada a traslaparse estratégicamente con la concepción esencialista de la diferencia cultural.

Desde la concepción cultural esencialista se entiende que es posible descubrir elementos característicos o esenciales de cada cultura, desconociendo la variabilidad interna inherente de los grupos culturales. A diferencia de la concepción étnica de la identidad denominada "constructivista», que concibe que la identidad es construida por los individuos y no se define objetivamente (Grammond 2014, 311-314) ${ }^{8}$. Es decir, parte de que la identidad no solo es algo que se hereda, sino algo que se construye. Por lo tanto, la identidad no puede ser algo estático, sólido e inmutable, considerándose la transculturación, la hibridación, el mestizaje como fenómenos propios del dinamismo cultural y no como patologías, desviaciones o corrupción de la cultura. Al respecto, Raquel Yrigoyen señala que:

«las culturas y formas de organización social se transforman y recrean constantemente; por tanto, hay muchos colectivos que tienen una identidad propia y diferenciada del resto de la sociedad nacional, con su propia cultura legal e instituciones, con herencia andina y también de otras fuentes, pero que ya no hablan idiomas indígenas ni mantienen elementos tradicionales que se consideran parte de una identidad indígena estereotipada» (Yrigoyen 2003, 178).

El esencialismo cultural tiene sus raíces en antiguos y estereotipados discursos sobre los indígenas. Rodofo Stavenhagen menciona que la creación de estas representaciones es de tipo colonial (Stavenhagen 2013, 36). En el imaginario social existe la idea del «indio puro», el

8 Esta postura sostiene que el discurso sobre la etnicidad es un artefacto que revela la tendencia de nuestra época a la exageración de las particularidades. 
que da continuidad a una línea de descendencia o cultura que debe permanecer intacta. Por ello, por ejemplo, el cambio del vestido, la declinación en el uso de la lengua o cambios lingüísticos, las transformaciones en la organización comunitaria o la migración a la ciudad $^{4}$ suelen ser interpretadas como un fenómeno de aculturación, menoscabándose su legitimidad como sujetos titulares de los derechos de la diferencia, poniéndola en crisis.

En definitiva, el marco regulatorio de los derechos de la diferencia establece el criterio de autodefinición cultural para determinar la pertenencia o no a un grupo indígena, esto es la conciencia de identidad, la misma que se encuentra asociada a las tradiciones y costumbres. Por ello se requiere que la identidad refleje, cuanto sea posible, las características esenciales de estos grupos. Sin embargo, tal como ha advertido el antropólogo Eduardo Restrepo 9 'la cultura' o 'diferencia cultural' de las poblaciones son 'culturalmente' producidas, generalmente desde posiciones hegemónicas, que no son percibidas como tales ya que hacen parte del sentido común de una época determinada (Restrepo 2004, 291).

La «autodefinición» se traslapa con la percepción dominante de los elementos culturales que debe poseer un grupo humano para ser indígena, subyace el mandato de «ser auténticos» y de cumplir diferentes elementos considerados esenciales de la cultura; y por ello en los documentos en los cuales se autodefinen (instrumentos constitutivos y reglamentos internos) o mediante los que se intenta regular la identidad, se invita a ensalzar la diferenciación y con ello a la exotización de la cultura, generando que la deje de ser para sí misma y se haga cultura para afuera. Los pueblos y sujetos indígenas ante la necesidad de conseguir un mayor reconocimiento de su participación deben estar continuamente legitimándose, etnizándose en ocasiones exotizándose como estrategia organizativa y reivindicadora frente al despojo, racismo e inequidades, lo que atenta al genuino dinamismo propio de la cultura, ya que se conmina a repetir la predefinida diferencia étnico cultural, anulándoles la posibilidad de que sus representaciones den cuenta de sus sentidos propios a la hora de pensarse a sí mismas. Las comunidades indígenas para garantizar la efectividad de la reivindicación necesitan resaltar atributos que les permiten mayores oportunidades, propiciándose un escenario que permite evaluar si los líderes y sus colectivos son lo suficientemente indígenas como para ser tales, reproduciendo el racismo y amenazando la reproducción genuina de la cultura. El hecho de que se

9 Sus investigaciones versan principalmente sobre el caso de las culturas negras. 
vean forzados a reproducir el discurso dominante de la diferencia genera que se cree la dicotomía del «indio con credenciales culturales» o «indio autorizado» y consecuentemente el «indio desautorizado», creándose un sur dentro del sur, excluyendo a quienes carecen de estructuras comunales, a quienes carecen de características visibles de la cultura o a quienes no cumplen el discurso dominante de lo que es ser indígena.

El reconocimiento cultural establecido en los derechos de la diferencia permite reivindicar derechos a la población indígena, pero en ocasiones no favorece y margina a determinados indígenas. La imposición de estereotipos y del discurso dominante sobre la identidad indígena genera un proceso excluyente en Ecuador. Entre los requisitos que establece la Subsecretaría de Interculturalidad de la Secretaría de Gestión de la Política para el reconocimiento de personería jurídica de colectivos indígenas se exige un acta de autodefinición, que la comunidad tenga mínimo 50 personas (en el caso de comunidades de la Sierra) 30 personas (en el Oriente) y límites demarcados. Es decir, en el imaginario burocrático, el ser indígena es incompatible con la ciudad y se define por estar vinculado a lo rural, a la selva, al campo. Merece reflexionar: ¿Qué sucedería con los indígenas urbanos, migrantes o desplazados que no se encuentren organizados en una delimitación territorial o con aquellas comunidades que se encuentren en disputa por el territorio? ¿Únicamente la asociación de territorio y cultura legitiman las reivindicaciones indígenas? ¿Es posible garantizar los derechos específicos de las personas indígenas urbanas que han sido desplazados por efectos de la minería o que carecen de estructura organizativa legalizada? (Los derechos relativos al territorio no tendrían cabida, pero sí otros derechos colectivos) ¿Pierden la calidad de titulares de derechos colectivos aquellas comunidades que no cumplen con el requisito que establece el número mínimo de personas? Estas exigencias pueden abrir una grieta en la que pueden caer personas que, considerándose indígenas, no pueden inscribirse, y por lo tanto impedir el ejercicio de sus derechos. Pensemos en algunos casos de personas indígenas que de forma individual o colectiva no cumplen los estereotipos que existe de este grupo humano: ¿es legítimo restringir la posibilidad de legalizar una colectividad de indígenas urbanos por no vivir en un territorio colectivo con límites demarcados? ¿Sería justo desconocer los derechos culturales de la población indígena que reside en la ciudad por haber sido desplazada a la ciudad por efecto de la minería (de estos últimos existen numerosos casos en el Ecuador)?

El objetivo de este análisis es llamar la atención sobre el debate de los derechos de la diferencia y generar una discusión crítica que contribuya a las luchas indígenas por sus derechos en el Ecuador. Es 
necesario que se extienda este marco analítico, poniendo en evidencia la complejidad de las lógicas presentes en el proceso de reivindicación de los derechos de la diferencia cultural para renovar los enfoques jurídicos y políticos, ya que tanto investigadores jurídicos como líderes indígenas nos encontramos enclaustrados en la reivindicación e investigación de los derechos culturales de la diferencia cuyas complejidades pueden renovar nuevas versiones colonizadas del proceso histórico de estas poblaciones, disminuyendo la capacidad de comprender los fenómenos sociales que se pretenden reivindicar. Amerita reflexionar si los derechos de la diferencia al corroborar la práctica de la esencialización permiten contestar al poder o, al contrario, lo reproducen. Este es un análisis que requiere ser abierto en Ecuador, principalmente en el campo de la investigación jurídica emancipatoria. Como ha advertido Rojas en su estudio sobre representaciones de afros en Colombia, la etnización da lugar a una paradójica forma de reconocimiento, en la que sólo es posible la igualdad de aquel que se asume como diferente; muchas veces sobre la base de los criterios que en un principio dieron lugar a su exclusión, o de aquellos definidos por los expertos como lo oficialmente diferente, es decir por quienes cumplen el modelo de alteridad oficial (Rojas 2004, 163).

En el Ecuador, pasada la época de proclamación y avance normativo realizado a partir de la constitución del 2008, es un momento propicio para detenerse a reflexionar sobre los límites inherentes a la reivindicación de los derechos culturales. Las complejidades que los acompañan invitan a examinar la concepción cultural que subyace a esta legislación; para ello es imprescindible vislumbrar las ataduras coloniales de los derechos contemporáneos de la diferencia cultural. Siguiendo este objetivo se recurrirá a ejemplos históricos como estrategia metodológica para entender el origen del esencialismo cultural en la identificación, ofreciendo un contexto histórico que permita explicar que el discurso jurídico en el intento de definir a la población actualmente denominada «indígena» ha creado categorías étnicas, políticas y jurídicas maleables que perviven en la actual interpretación esencialista de la legislación. Se citarán dos casos históricos que generan insumos de discusión sobre los criterios para identificar y denominar a la población «indígena» y explicar que cualquiera que sea el método o criterio que se utilice para definirlos e identificarlos, ya sea de asignación externa o autodefinición, se encuentra supeditado al modelo de alteridad oficial, a la subjetividad y esencialismo (racial, genealógico o cultural) de la interpretación y percepción de quien otorga los derechos. 
En virtud de que interesa la perspectiva gubernamental, se revisarán los conceptos y categorías que existían sobre los indígenas en el discurso jurídico colonial y global. Para ello se citará un litigio colonial analizado por la historiadora Nancy E. Van Deusen, por ser el caso más ilustrativo en el cual la determinación de la libertad o esclavitud de los entonces denominados «indios» dependía de la evaluación de identidad que realizaba la burocracia colonial. Dado el especial interés por los procesos de definición e identificación a la población indígena en Ecuador, se realizará un acercamiento a la historia del primer censo en el Ecuador (1950) en virtud de que ilustra la ineficacia de evaluar esencialmente las características culturales para categorizar a la población indígena.

Previo a este análisis, partamos de la premisa de que la identidad posee un carácter construido, es decir, es el producto de la interacción social, no existe la identidad "auténtica». Los atributos culturales no son fijos, la identidad puede ser múltiple, matizada, ambigua, se puede tener una o más identificaciones.

\section{Revisión sumaria de la categorización e identificación de la población indígena durante la colonia}

La colonización es una de las etapas de la historia que marcó trascendentalmente la historia de América y del actual Ecuador. El proceso de conquista fue territorial, político, ideológico, cultural y social. Fue una guerra amparada en el paraguas jurídico-religioso del derecho hispano; durante la época colonial la población indígena estuvo sujeta al trabajo obligatorio y la evangelización, lo cual condujo no solo al despojo de sus tierras, sino al inicio de una serie de transformaciones culturales y sociales dentro de las comunidades indígenas. Esta época histórica en el Ecuador ha dejado hasta la actualidad sesgos de colonialidad.

Según Aníbal Quijano, no solo se trata de imaginarios o discursos que se traducen en "aparatos» de poder y patrones disciplinarios (leyes, instituciones, burocracias coloniales), sino también en formas concretas de subjetividad, modos de vida, estructuras de pensamiento y acción incorporadas al habitus de los distintos grupos sociales (Agenda Nacional Para La Igualdad de Nacionalidades y Pueblos 2013-2017, 10).

Para tener una visión panorámica de los regímenes jurídicos que administraban a los denominados «indios» durante la etapa colonial se partirá de la división en dos períodos que dan cuenta de la concepción y condición de su capacidad jurídica en el derecho indiano. 


\subsection{Primera etapa}

En el discurso jurídico a partir de finales del s. XVI (1492-1511) el denominado «indio» fue clasificado como un ser inferior por su irracionalidad, prácticas sanguinarias, considerándolo un objeto, con lo cual se justificó la expansión colonialista. Para la época eran considerados como amentes (Rodríguez 1994, 315).

\subsection{Segunda etapa}

Posteriormente, a partir del establecimiento del decreto denominado veritas ipse (1537) que proclamó los principios para el buen gobierno de las indias, se consideró la condición humana del indio americano (Icaza Dufour 1992, 107). Se reconoció la naturaleza jurídica de «libre» pero como «vasallo» debía trabajar para la corona, debían ocuparse con ejercicios corporales o espirituales, a fin de que no recayeran en la idolatría, dando lugar a la encomienda. Es decir, se concebía al indio libre con la capacidad de un niño.

Si bien este decreto generó un cambio en la polémica legal, la esclavitud pervivía en la práctica, por ello el debate no terminó con este decreto. Posteriormente se profundizó sobre la misma cuestión en la conocida "polémica de los naturales o justos títulos» que la Junta de Valladolid legalizó a través de la expedición de las Leyes Nuevas en 1542, que determinan que no existe ninguna causa que permita esclavizar a un indio. Expedidos estos decretos, en 1550 se suscitó el conocido diálogo de Valladolid, en el cual se discutieron los derechos de los naturales del Nuevo Mundo y la legitimidad de la conquista. A pesar de que discursivamente los indios eran libres, se les consideraba como sujetos con incapacidad relativa. Eran equiparados con «los españoles rústicos y miserables», sometiéndolos a un régimen de tutela y protección en sus relaciones con los españoles y con las castas (Cruz Barney 2012, 325). Finalmente, con la expedición de la Constitución de Cádiz se introdujo una noción teórica de igualdad entre españoles, mestizos e indios, que en la práctica no conllevó ningún cambio significativo para esta población.

Esta mención al discurso jurídico mediante el cual se establecían los "derechos de los indios» en la etapa colonial da cuenta de los estereotipos e imaginarios que justificaban su dominación. Aparentemente la clasificación racial, denominada «las castas», era un sistema rígido de diferenciación étnica, con la cual se determinaba la condición y capacidad jurídica de las personas pertenecientes a las 
distintas «razas». Sin embargo, este sistema fue complejo ya que las características esenciales «raciales» de cada jerarquía de las castas eran absolutamente variables y difusas. Historiadoras de la etapa colonial, como Nancy E. Van Deusen y Joanne Rappaport, han demostrado las dificultades que existían para determinar quiénes pertenecían a la categoría «indio» (Van Deusen 2015, 190). La subjetividad de la asignación de la «raza» puede verse plasmada en los documentos de compra y venta de los esclavos, donde una misma persona podía constar como «indio» en un documento y «mulato» en otro. El sistema de castas no era un sistema rígido de clasificación; al contrario, era difuso.

Con la finalidad de identificar a los «indios», un perito conocedor de las indias y sus habitantes era llamado a escrutar visualmente a los litigantes para determinar el aspecto, cualidades y características que podían ayudar a identificarlos. El método de identificación se basaba en la observación de movimientos, forma de la cabeza y cara, color, expresiones faciales, cabello, tipo de piel, gestos, comportamiento, estructura corporal (Van Deusen 2015, 176). Además se evaluaba su lengua, religión, nacionalidad o su linaje. Se utilizaba el criterio racial genealógico y la fisonomía. Con el fin de comprender la elasticidad y manipulación a la que daba lugar este método revisemos el litigio de libertad del Perafán de Rivera vs. Pedro (cuyo apellido no se informa), caso analizado por la historiadora Nancy E. Van Deusen.

\subsubsection{Caso del Comendador Perafán de Rivera vs. Pedro}

Recordemos que la esclavitud de los indios de América estaba prohibida oficialmente desde 1530, aunque se practicaba ilegalmente. En 1560, en la Sala de Justicia del Consejo de Indias, se litigó la libertad del esclavo Pedro en oposición al Comendador de Toledo, Perafán de Rivera. Pedro afirmaba que era originario del Virreinato de Perú. El Comendador interpeló esta afirmación y presentó como evidencia dos tickets de su venta que demostraban lo contrario. En el primer ticket de venta de Pedro se le había registrado como un «loro» (color café verdoso, distinto al negro) capturado en guerra justa; el segundo ticket lo identificaba como un "negro» de talla media con una cadena de hierro en su cuello. Ningún ticket de venta especificaba el origen geográfico de Pedro. El abogado de Pedro y el testigo Fray Francisco de Carvajal argumentaron que los indios de América no eran negros, por lo tanto, no podían ser esclavos. La categoría «negro» solía ser utilizada estratégicamente. Portugueses y comerciantes utilizaban la categoría 
negro para identificar y esclavizar. Por ello se solía «ennegrecer» a los indios de América en los documentos y así legalizar su esclavitud.

Para argumentar que Pedro era indio de América $-\mathrm{y}$ por lo tanto libre- Fray Francisco de Carvajal testificó que la primera vez que vio a Pedro dijo: "Ven acá, hermano indio, ¿por qué tienes esas cadenas y candados de hierro si tú eres un indio y libre por tu naturaleza?». Describió a Pedro con pelo y barba muy negros, pero no tan rizado como el de los negros y mulatos. Su explicación fue crucial al describir que el color de la cara de Pedro era más oscuro, porque «las caras de los indios de la costa son más tostadas». Con ello, relacionaba geográficamente el color de piel de Pedro con la costa de América y no con la de África. La identidad negra o india de Pedro iba a ser determinada entonces por su lugar geográfico de origen, si era un indio de América no podía ser esclavo.

El esclavo Pedro podía obtener la libertad si demostraba que su coloración «tostada» era de las costas de América y no de África, quedando su evaluación e identificación al arbitrio de la percepción y subjetividad de los peritos, jueces e intereses de los comerciantes. Esta historia da cuenta que las características físicas asociadas a los grupos raciales no eran evidentes, al contrario, eran maleables y estaban sujetas a la subjetividad de la burocracia colonial. Además, existían otros componentes - como el lugar de origen en este casoque también entraban en la disputa para construir, usar y manipular las categorías raciales y jurídicas al interior de las cortes coloniales, visibilizando que estas eran inestables, indefinidas, podían ser alteradas, maleables y eran susceptibles de manipulación.

\section{Revisión sumaria de la normativa ecuatoriana relativa a los pueblos indígenas}

Previo a realizar el análisis del censo ecuatoriano de 1950, contextualicemos brevemente el camino recorrido relativo a los derechos de los pueblos indígenas en el Ecuador.

Las nuevas repúblicas de América Latina del siglo xx definieron a las colectividades indígenas como grupos marginalizados y atrasados, cuya misma existencia amenazaba el orden social y el progreso. En tal razón se inició el proceso de expandir el modelo homogeneizante, en palabras de Yrigoyen:

Se instauró el modelo de Estado Nación, con una sola cultura, un solo idioma y una sola religión oficial, que se plasmó en la 
Constitución. Se buscaba la asimilación o desaparición de la cultura indígena dentro de la nación mestiza, bajo la ideología del progreso y la superación del «atraso indígena». Se buscó extinguir los idiomas indígenas, su religión, su cultura (Yrigoyen 1999, 47).

Se puede colegir que la instauración de este modelo hegemónico empujó a los pueblos indígenas en dos direcciones divergentes. Podrían asimilarse dentro del nuevo orden nacional, pero solo a costa de renunciar a sus propias prácticas e identidades culturales. Si optaron por rechazar el nuevo orden de sociedad, se los consideraría como salvajes, feroces e infrahumanos. La historia republicana del Ecuador da cuenta de varias «liberaciones», "revoluciones» y la emergencia constante de grupos contestatarios (revolución marxista de 1845, revolución liberal de 1895, revolución juliana de 1925). Sin embargo, en ninguna de estas coyunturas existió una verdadera reivindicación hacia los intereses de la población indígena. Durante la vida republicana del Ecuador, este grupo humano ha sido sometido y despojado de su trabajo, territorio y de humanidad.

El modelo de Estado que se instauró desde inicios de la República (1830), proclamaba al Ecuador como un Estado monocultural, adoptándose la teoría liberal que concibe al Estado como una igualdad formal de todos los habitantes ante la ley. Es decir, que se configura un modelo de Estado cuya cultura nacional es la blanca mestiza, una sola forma de autoridad, una sola forma de producción del derecho desde el denominado poder legislativo, una sola forma de administración de justicia y el monopolio del uso de la fuerza. El surgimiento del Estado nación significó para la población indígena un sometimiento a la cultura dominante, también denominada modelo de asimilación (Jaramillo 2011, 51).

Posteriormente, se inicia el modelo político de integración en el que se amplía la cobertura de derechos a los individuos de la población indígena, reconociéndoles únicamente sus derechos individuales, como es el ejercicio de su ciudadanía (1857). De esta manera se integran en el modelo nacional, asumiendo implícitamente su condición de ciudadanos, pero esta definición no era operativa para fines electorales, ya que a esta población se le restringió el derecho al voto por ser analfabeta (Illaquiche 2001). Recién con la expedición de la Constitución de 1978, al eliminarse el requisito de saber leer y escribir, adquirieron derecho al voto. Bajo este modelo de integración inicial se consideraba a los indígenas como menores permanentes; es decir, estaban concebidos como incapaces de administrarse por sí mismos, y por tanto necesitaban de tutores o de guardianes, vislumbrándose 
entonces una continuidad del mencionado decreto colonial, veritas ipse de 1537 que concebía al «indio libre» con la capacidad de un niño.

En el discurso gubernamental ecuatoriano se ha pasado por pensar al indígena como una carga para el Estado (raza que debe ser mejorada) a pensarlo como «pobre», creándose en el mejor de los casos políticas para la atención de la pobreza, con un carácter paternalista e integracionista que desempoderaba y desestructuraba a esta población. En los ochenta, con el proyecto del indigenismo legal que se desplegó a nivel latinoamericano, la política fue dirigida ya no a integrar a la población indígena a través del mecanismo de la asimilación, sino a incorporar a la población indígena como tal al nuevo proyecto de Estado, incorporándose algo del lenguaje de los derechos humanos. De acuerdo con Rodolfo Stavenhagen el movimiento indígena inició con demandas por la redistribución y justicia histórica, con una gran idealización del pasado. Durante los setentas y principios de los ochentas las demandas se centran en la solución de conflictos agrarios y de bienestar social. Durante este periodo, los reclamos por justicia económica y social fueron acompañados por demandas de reconocimiento legal de autonomía y autodeterminación, en donde la identidad étnica se convirtió en el principio unificador (Díaz-Polanco 1991).

De la revisión de la historia jurídica republicana hasta los noventas se colige que la situación de los pueblos indígenas ha sido la de «indígenas entre constituciones mestizas», tal como ha titulado Bartolomé Clavero en su libro publicado en el 2008. Uno de los acontecimientos claves para poner en discusión los derechos indígenas fue el levantamiento indígena de los noventa, que en el Ecuador vino acompañado por la ratificación del Convenio 169 de la OIT (1998), el cual fue relativamente recogido en la Constitución de 1998.

Este acontecimiento que merece especial atención para comprender la situación actual sobre la autoidentificación en el Ecuador fue el detonante para fracturar la visión monocultural del Ecuador, marcando el inicio de un proceso orientado a la liberación de los pueblos indígenas. Este acontecimiento representa el inicio del tránsito de una visión racializada y fisonómica de lo indígena, al reconocimiento de culturas diferentes. Su reivindicación se desarrolla en el marco de la recuperación o reafirmación de sus identidades (Ortiz 2011, 68). Si bien el movimiento indígena y las luchas indígenas, como tal, datan de épocas coloniales, este levantamiento es considerado como el antecedente inmediato para el nacimiento de un movimiento indígena estructurado en todos los niveles (Larrea 2004, 97). Se mostró no solamente la existencia de un Ecuador profundo, con pueblos olvidados y excluidos, sino que además planteó serios cuestionamientos a un 
modelo de democracia absolutamente excluyente en el que los pueblos indígenas no tenían cabida y con un modelo de desarrollo construido sobre ellos, de espaldas a ellos y sin ellos. (Larrea 2004, 11-15). Las consecuencias jurídicas de este levantamiento y del subsiguiente empoderamiento del movimiento indígena se han traducido en reformas normativas en las constituciones de 1998 y 2008. Dado el avance normativo de la última constitución, esta revisión quedaría incompleta si no se realiza una mención a la situación actual en el Ecuador sobre los derechos culturales. A pesar del despliegue de derechos culturales y de autodeterminación de los pueblos indígenas, en la última década los mismos también han sido utilizados para dividir y neutralizar a los movimientos indígenas (se ha vulnerado especialmente el derecho a la consulta previa). Podría decirse que los derechos culturales han sido más o menos respetados en tanto no amenacen la integridad del sistema extractivista. El discurso gubernamental que proclama la necesidad de valorar a las culturas indígenas, sus conocimientos ancestrales, lengua, estética, en cierta medida ha desenfocado los problemas de fondo: despojo de territorios indígenas y la afectación minera.

\subsection{El primer censo ecuatoriano (1950)}

Una vez reseñada la historia jurídica del Ecuador con respecto a los derechos de la población indígena, se hará referencia a un acontecimiento específico de la historia ecuatoriana de mediados del siglo Xx. Para ello revisaremos el análisis de la historiadora Laura Giraudo quien ha reconstruido el recorrido de la institucionalización de la estadística en América Latina. En 1940 hubo un intento del sector del indigenismo estatal por conseguir un criterio uniforme y científico de definición de los indígenas para todo el continente americano (Giraudo y Bustamante 2014, 129). En el acta del Primer Congreso Indigenista Interamericano (1940) constan las recomendaciones de incluir en las estadísticas las características culturales de la población "sobre una base de comparabilidad interamericana», sugiriendo que se usen las características culturales para clasificar a los grupos de población (Giraudo y Bustamante 2014, 145). En el Ecuador se realizó por primera vez un censo nacional en 1950, en cumplimiento con el compromiso de participar en el Censo Continental de 1950 (Giraudo y Bustamante 2014, 129, 145, 174). La posición oficial de la Dirección de Estadística del Ecuador fue la de no incluir pregunta acerca de raza o color, ni acerca del idioma, religión y propiedad 
(Comentarios para el censo de las Américas en Giraudo y Bustamante 2014 , 176) para el censo de 1950, en el cual se plasmaron conceptos de higiene, economía, biología y cultural, agrupando características socio económicas y/o elementos culturales para definir al indígena.

A manera de ensayo previo al censo de 1950, se realizó en 1947 un censo urbano en Quito. En este caso, los encuestadores debían anotar sus observaciones acerca de la raza, pero fueron explícitamente instruidos para que no preguntaran la «raza» a los habitantes, sino que anotaran una categoría como resultado de sus observaciones. Para guiarse en esta tarea se sugería tomar en cuenta el uso de trajes típicos indígenas, el pelo largo en el caso de los hombres, las "formas de expresión» y el escaso conocimiento del español (Clark, citado en Giraudo y Bustamante 2014, 177), es decir, definirles a través de características culturales. Es interesante que, si bien esta fue la posición oficial de la Dirección de Estadística, no todos la compartían.

El antropólogo Antonio Santiana escribió acerca de la importancia de la clasificación racial del indio, sosteniendo que lo ideal sería practicar en cada caso un «diagnóstico racial», que incluyera desde los índices cefálicos hasta los grupos sanguíneos. Sin embargo, reflexionaba que la realización de este diagnóstico individual era irrealizable por la imposibilidad de establecer una separación radical entre una raza y otra. Por ello, afirmó que no quedaba otro camino que las características de orden cultural, también susceptible de error por la tendencia a calcular como mestizos a indios que viven en la ciudad, visten como blancos pobres o hablan castellano. Además, señala que los que han vivido cerca del «indio» podrían tener cierta insensibilidad ante sus rasgos físicos, así que sugiere que sean europeos, poco familiarizados con el indio, quienes realicen el recuento en las ciudades (Giraudo y Bustamante 2014, 177).

En el censo nacional de 1950 no se incluyó una categoría censal para identificar a los indígenas y se intentó definirlos a través de sus características culturales para cuantificar a esta población, dado que se asociaba la «raza» a un determinado estado socioeconómico y cultural, con referencias a la vivienda, calzado y cama. Sin embargo, los resultados no permitían hacer tal clasificación ya que, según el elemento que se considerara más adecuado para indicar la indianidad, la población indígena podía variar entonces desde una minoría hasta una gran mayoría de la población total. Por ejemplo: en Loja, el 26\% vivía en choza, el 33\% andaba descalzo, el $40 \%$ usaba osotas, pero sólo el 3,5\% hablaba quichua. En Bolívar y en Azuay, el $49 \%$ y el $27 \%$ respectivamente vivían en choza, pero un porcentaje mucho más alto andaban descalzos (74 y 61\%) (J. Van 
D. Saunders, citando en Giraudo y Bustamante 2014, 37). Según el elemento que se considerara la población indígena podía variar entonces desde una minoría hasta una gran mayoría de la población total. Los resultados reproducían la percepción dominante acerca de los indígenas, definidos por sus condiciones de vida —elementos culturales- (Giraudo y Bustamante 2014, 179). La imagen de la población indígena del Ecuador de 1950 se construyó entrelazando los siguientes resultados: el $11 \%$ era hablante quechua, el $40 \%$ vivía en chozas, el $50 \%$ andaba descalzo, el $22 \%$ dormía en el suelo y el $27 \%$ dormía en tarima o hamaca.

Tanto en el censo de Quito de 1947, en el cual un tercero evaluó la supuesta raza, como en el censo de 1950, la población indígena se define a través de elementos socio-culturales (Giraudo y Bustamante 2014, 177). Los resultados de este censo (1950) reflejaron caóticamente la percepción dominante de los elementos culturales de la población, mas no permitía una clasificación estricta. Esta experiencia clasificatoria basada en los imaginarios creados por un sector del indigenismo oficial da cuenta de la inutilidad de objetivar a partir de la atención estricta a los elementos culturales con los cuales se pretendía identificar a la población. Este intento clasificatorio, basado en las características culturales, medios económicos, condiciones de vida y con explicaciones de las diferencias culturales de los indígenas, presentó complejidades concretas al momento de cuantificar a este sector de la población $y$, en definitiva, no cumplió el objetivo de identificar con certeza el número de individuos indígenas.

Antes de dar las últimas puntadas a este documento y elaborar las conclusiones, es necesario hacer una referencia a la autoidentificación indígena en el Ecuador y al método de identificación del último censo de población y vivienda (2010). Para esta visibilización estadística que constituye la fuente para la elaboración de políticas públicas se han establecido categorías oficiales étnicas, políticas, culturales e incluso fisonómicas o de pigmento-identificación, ${ }^{10}$ utilizándose las siguientes categorías: indígena, afroecuatoriano, negro, mulato, montubio, mestizo, blanco. Así mismo, la identificación indígena se desagrega en catorce nacionalidades y en dieciocho pueblos indígenas.

10 Término acuñado por activistas afroecuatorianos para referirse a la identificación según el color de la piel. Así mismo se utiliza el término "pigmentocracia» para referirse a la desigualdad de oportunidades que se generan a partir de la supremacía del color «blanco» de la piel. 
Los resultados del censo dan cuenta de que el 7,03\% de la población ecuatoriana se autoidentifica como indígena (CODENPE, 2012), esto motiva la creación de las imprescindibles políticas públicas interculturales, aunque simultáneamente ha servido para desconocer su importancia en tanto esta identidad es concebida como una «minoría» ya que, a simple vista estadística, las cifras indican que la población indígena representa solo el 7\% frente al $71,9 \%$ de mestizos. ${ }^{11}$

Indudablemente estas mediciones tienen varios límites, entre los principales la ideología del «mestizaje» impregnada del concepto de «blanqueamiento»12. Así mismo, es probable que la poca tendencia a declararse como «indígena» en el censo se deba a que esta identidad, a pesar de que se encuentra revalorizándose en base al discurso de los derechos de la diferencia cultural, simultáneamente sigue siendo una identidad rechazada y estigmatizada.

\section{Conclusiones}

Revisados los insumos históricos referenciados se puede deducir que se traza cierta circularidad en la historia en el criterio de identificación de la población indígena. En distintas épocas y escenarios se crearon conceptos clasificatorios para determinar su estatus jurídico. El ser «indio» o «indígena» es una categoría de poder que ha sido construida, deconstruida y transformada históricamente para administrarlos. La maleabilidad de la categoría colonial «indio», citada en el litigio sobre la libertad de Pedro Farfán, ejemplifica la manera en la que el derecho realiza fijaciones, construyendo abstracciones que a la hora de ser interpretadas permitían polivalencia del concepto de lo que es ser «indio» y de su uso. El derecho en búsqueda de la certidumbre ha pretendido traducir jurídicamente la identidad indígena a través de la genealogía, evaluación racial, fisonómica, cultural, evaluando la identidad a través de la percepción de la mirada

11 En contraste con la diferencia de la estadística que se presumía a fines del siglo XVIII, que indicaba que la población de «indios» era una mayoría que representaba el $68,44 \%$ y la de mestizos apenas representaba el $4 \%$ (Ayala Mora, citado en Agenda Nacional Para La Igualdad de Nacionalidades y Pueblos 2013-2017, 10).

12 El «mestizaje» como categoría étnica dominante del Estado nación en el Ecuador ha sido ampliamente investigada por Kingman, Erika Silva, Catherine Walsh, o Andrés Guerrero (2010), entre otros investigadores. 
oficial. Generalmente las abstracciones y formalizaciones legales en búsqueda de la certidumbre son restrictivas e inflexibles, lo cual es incompatible con la naturaleza cambiante de la cultura.

Los resultados del intento de construir la categoría «indígena» mediante el censo de 1950 fueron difusos y también quedaban supeditados al arbitrio de una mirada escrutadora que, al igual que en la colonia, participaba en el reconocimiento e identificación, en este caso a partir de la descripción de la vestimenta, la vivienda o la lengua, entre otras características.

Se ha evidenciado que la clasificación de los grupos humanos en distintos órdenes jerarquizados mediante categorizaciones genealógicas, raciales y culturales, lejos de ser simple y rígida, ha sido maleable. Los dos datos históricos permiten comprender que, sin importar cuál sea el método o criterio que se utilice para definir e identificar a los indígenas, se encuentran supeditados a la subjetividad y esencialismo de la interpretación y percepción de quien los reconoce desde sus parámetros y referencias, poniendo de relieve la subjetividad en el intento de conseguir conceptos que lo definan. Pese a ello, se intenta objetivar lo que por naturaleza es cambiante, exigiendo que las culturas sean «puras» e «intactas», con diferencias esenciales que suelen asociarse a estereotipos oficiales que tienen un parentesco con los imaginarios y premisas del criterio fisonómico colonial y con el criterio del cumplimiento de las características culturales que se observan del censo de 1950.

Se ha puesto de relieve las dificultades que se suscitan en el seno del discurso jurídico de los derechos de los pueblos indígenas, exponiendo la amenaza que supone a las culturas el esencialismo cultural, la presión que genera la percepción dominante sobre la cultura.

En el discurso gubernamental sobre la identidad indígena palpita el imaginario colonial sobre los «indios». Este discurso aboca al indígena a reconocerse a partir de significados dominantes basados en discursos sobre la identidad. La exigencia o presión estatal de repetición y continuidad con el pasado genera la necesidad forzosa de los pueblos indígenas a "conservarse» como estrategia para asegurar sus demandas, ya que existe la necesidad de inscribir la autodefinición según el mandato de la percepción dominante con el objetivo de reafirmar la identidad para reivindicar derechos, titular sus tierras y contribuir al proyecto de interculturalidad.

Asimismo las normativas nacionales destinadas a proteger la diferencia, regulando la salud, educación intercultural, patrimonio, propiedad intelectual, suelen utilizar los verbos rectores «preservar 
y conservar la cultura», revelando una visión conservadora de que la cultura debe permanecer intacta, vaciándola de contenido, cosificándola y amenazando a su viva reproducción; la cultura es un producto de la interacción social. Como ha señalado Grammond, el esencialismo y exotización de la cultura es un problema que no debe ser subestimado, ya que si una cultura se plasma como inmutable puede acabar negando la identidad de los individuos que adoptan un comportamiento juzgado como «no tradicional», oficializando visiones puramente estereotipadas de la cultura (Grammond 2014, 318).

Las sociedades son complejas, varios pueblos indígenas además se encuentran entre el conflicto del deseo de conservarse y el de adaptación a las necesidades de la modernidad, por ello necesariamente una autodefinición tendría que ser referencial, cambiante, al interior del grupo.

Un riesgo que tiene este análisis es que se desvíe o se malinterprete, la intención es detenerse a reflexionar sobre la exotización, el esencialismo cultural y el modelo de alteridad oficial indígena. La preocupación principal de este análisis es el control político de la diferencia; es decir, que estos problemas culturales se relacionan con otros directamente políticos, ya que de lo analizado se puede deducir la categoría del «indígena autorizado» que resulta ser el indígena del imaginario del blanco mestizo que cumple el modelo referente de la alteridad oficial o plasma características culturales visibles (vive, danza, viste multicolormente), en oposición al «indígena desautorizado», subalternizado, que habita en la marginalidad urbana o en el sector rural, sin límites demarcados o que no cumple plenamente la expectativa hegemónica para merecer derechos culturales diferenciados.

Súmese a esto, las complejidades descritas sobre los requisitos solicitados por el Estado ecuatoriano para legalizar un colectivo (límites demarcados, número de personas adscritas), que dan lugar al replanteamiento de la premisa jurídica que establece la asociación de territorio originario e identidad cultural como fuente legítima para la reivindicación de sus derechos ${ }^{13}$.

La concepción de lo que es ser indígena, en el marco de la legislación internacional relativa a pueblos indígenas y de la legislación

13 El camino para cuestionar esta premisa ha sido abierto por el investigador Mikel Berraondo (2009), quien ha planteado las implicaciones del Estado español por la ratificación del Convenio 169, ya que este instrumento sería aplicable a la población indígena en territorio español (especificando que existirían reservas y matices). 
ecuatoriana, parte de fijaciones que desde antaño han sido polisémicas, difusas, ambivalentes y han estado lejos de cualquier comprobación empírica. Por ello se concluye que no es posible buscar respuestas rígidas, taxativas o estáticas para identificación indígena, reconociendo que toda identidad se encuentra en constante actualización, es dúctil, dinámica, y que las transformaciones de la cultura no constituyen un abandono de la identidad.

Si bien en el discurso jurídico contemporáneo el criterio de la autoidentificación se presenta como el método más abierto, podríamos considerar que pervive el sistema clasificatorio basado en los elementos culturales con el modelo de la autoidentificación. En definitiva, el esencialismo racial y genealógico que originalmente fue la piedra angular del derecho indiano, actualmente es cultural. La historia se nos presenta como un espejo, la legislación contemporánea es heredera de los imaginarios construidos de antaño, el criterio de autoidentificación se nutre del pasado medieval y no escapa de la asignación externa, no solo basta ser, hay que parecer indígena, ya que el poder define, persiste la mirada y representación colonial en los elementos que forman el concepto de indígena. El criterio de autoidentificación y el de criterios culturales conviven.

Los derechos culturales contemporáneos poseen una potencialidad emancipatoria, contribuyendo al proyecto intercultural y de equidad. Es indudable que el paso del criterio censal de identificación mediante los evaluadores de indígenas al criterio de autodefinición fue un avance, pues la autodefinición puede erigirse frente a la imposición cultural hegemónica, pero simultáneamente los derechos consecuentes de la diferencia pueden ser interpretados e instrumentalizados para deslegitimar estratégicamente a las comunidades, es decir, para el control político de la diferencia.

En definitiva, la legislación sobre derechos de los pueblos indígenas, por una parte, puede ser usada estratégicamente por sus titulares; por otra parte, supone simultáneamente dificultades tanto al interior de las comunidades (exotización) como fuera de ellas, ya que la materialización de estos derechos pasa por la interpretación de los operadores jurídicos del Estado (esencialización). Se produce una paradoja pues la esencialización permite la resistencia y simultáneamente padece de una atadura del discurso dominante al cual se opone y disputa. Además el condicionamiento de esencialización de los derechos de la diferencia puede inclusive conllevar la negación de otros rasgos culturales o referentes identitarios, alentando a que difiera la identidad vivida de la identidad legalizada. 
Este argumento no debe ser leído como crítica a la agencia política indígena, sino principalmente, como crítica a las nuevas formas de gobierno que limitan y hasta deforman tales estrategias; un nuevo racismo institucional, con cara pluralista y hasta simpático, que bloquea, con más eficacia aún, los anhelos libertadores del pueblo indígena en su conjunto. (Hale 2004, 8). Es para llamar la atención a la tendencia a generar una dicotomía entre los indígenas que cumplen la diferencia del modelo de alteridad. Es decir, entre quienes «merecen» los derechos culturales y los que no están en condiciones de gozarlos a cabalidad. No se trata de una crítica a los derechos de la diferencia cultural en sí, sino a las consecuencias. La manera que en se abren espacios de participación política indígena al mismo tiempo genera límites para sus aspiraciones de transformación estructural. Por una parte, se reivindican varios derechos y se crean oportunidades políticas, pero la supremacía cultural se sigue reproduciendo, en virtud de que se ven obligados a reproducir el discurso dominante que se reproduce sobre ellos, conllevando una racialización que puede ser directa o sutil o solapada. Incluso los «indígenas autorizados», si bien ocupan cargos en la "etnoburocracia", los mismos se encuentran en los escalones más bajos de la jerarquía laboral. Adicionalmente, esta presión colabora en la interiorización de las normas raciales de dominación.

Para terminar, amerita una reflexión adicional final sobre la importancia no solo de reafirmar las diferencias, sino de aprender a convivir y reconocer sus transiciones y transformaciones históricas, hibridaciones, sobrepasando el pensamiento binario que califica de aculturación a las dinámicas del intercambio cultural y a las estrategias para convivir con las exigencias de la modernidad. En este sentido se requiere que las políticas culturales estatales garanticen la construcción y afirmación identitaria sin desconocer el dinamismo y la mutabilidad de las culturas. Es necesario deconstruir las formas estereotipadas de ser indígena y reconocer la existencia de formas de ser indígena que han sido subalternizadas por no cumplir el modelo de alteridad oficial, socavando aquellas formas desautorizadas de serlo.

\section{Bibliografía}

Agenda Nacional Para la Igualdad de Nacionalidades y Pueblos. Realizada por el CODAE, CODEPMOC, CODENPE, con el apoyo técnico de Senplades. 2013-2017.

Berraondo, Mikel. 2009. La Ratificación del Convenio 169 de la OIT por España. Reflexiones en torno a sus implicaciones, Madrid: AECID. 
CODENPE. 2012. VII Censo de Población y VI de Vivienda 2010. Quito: INEC, CONEPIA

Cruz Barney, Oscar. (2012). Historia del derecho indiano. Valencia: Tirant lo Blanch.

Díaz-Polanco, Héctor. 1991. «Etnias, Modernidad y Autonomía». En Conciencia Étnica y Modernidad editado por Edna Maria Orozco. México: INI.

Giraudo, Laura. 2007. «Entre rupturas y retornos: la nueva cuestión indígena en América Latina». En Ciudadanía y derechos indígenas en América Latina: poblaciones, estados y orden internacional, Bartolomé Clavero, et al., 7-57, Madrid: Centro de Estudios Políticos y Constitucionales.

Giraudo, Laura y Jesús Bustamante. 2014. La novedad estadística. Cuantificar, cualificar y transformar las poblaciones en Europa y América Latina, siglos XIX y XX, Madrid: Ediciones Polifemo.

Grammond, Sebastian. 2014. La Identidad Indígena Apropiada por el Derecho en Los Puentes entre la Antropología y el Derecho. Orientaciones desde la Antropología Jurídica. Chile: Programa de Antropología Jurídica e Interculturalidad, Facultad de Derecho, Universidad de Chile.

Guerrero, Andrés. 2010. Administración de poblaciones, ventriloquía y transescritura. Análisis históricos: estudios teóricos. Lima: IEP; FlacsoEcuador.

Hale Charles. 2004. El protagonismo indígena, las políticas estatales y el nuevo racismo en la época del «indio permitido». Ponencia para la conferencia, "Construyendo la paz: Guatemala desde un enfoque comparado», organizada por la Misión de Verificación de las Naciones Unidas en Guatemala (MINUGUA), 27-29 de octubre.

Illaquiche, Raul. 2001. "Ciudadanía y Pueblos Indígenas». Fecha de acceso: 20 octubre 2018. Disponible en: http://icci.nativeweb.org/boletin/22/ Ilaquiche.html

Jaramillo, Marcel. 2011. El nuevo modelo de Estado en el Ecuador: Del Estado de Derecho al Estado Constitucional de Derechos y Justicia. Quito: Universidad San Francisco de Quito, Fecha de acceso: 20 octubre de 2018. Disponible en: http://repositorio.usfq.edu.ec/bitstream/23000/668/1/99788.pdf

Larrea, Carlos (coordinador). 2004. Pueblos indígenas. Desarrollo Humano y Discriminación en el Ecuador. Quito: Abya-Yala y Universidad Andina.

Restrepo, Eduardo. 2004. «Biopolítica y alteridad: dilemas de la etnización de las colombias negras». En Conflicto e (in) visibilidad. Retos en los estudios de la gente negra en Colombia editado por Restrepo, Eduardo y Axel Rojas, 271-300. Colombia: Editorial Universidad del Cauca.

Rodríguez, Antonio Dougnac. 1994. Manual de historia del derecho indiano. México, D.F.: Universidad Nacional Autónoma de México.

Rojas, Axel. 2004. "Subalternos entre los subalternos: presencia e invisibilidad de la población negra en los imaginarios teóricos y sociales». En Conflicto e (in) visibilidad. Retos en los estudios de la gente negra en Colombia. 157-173, Colombia: Editorial Universidad del Cauca.

Rodríguez, Antonio Dougnac. 1994. Manual de historia del derecho indiano. México, D.F.: Universidad Nacional Autónoma de México. 
Sánchez, Pedro. 2013. ¿Qué significa ser indígena para el indígena? Más allá de la comunidad y la lengua. Quito: Abya Yala.

Stavenhagen, Rodolfo. 2010. Los pueblos originarios: el debate necesario. Buenos Aires: Clacso.

Stavenhagen, Rodolfo. 2013. "Pueblos Indígenas: retos después de la batalla». En Los derechos indígenas tras la Declaración. El Desafío de la implementación editado por Felipe Gómez Isa y Mikel Berraondo, 15-39, Bilbao: Universidad de Deusto.

Van Deusen, Nancy. 2015. Global Indios: The indigenous Struggle for Justice in Sixteenth - Century Spain. Durham, N.C.: Duke University Press.

Ventura, Monserrath. 1997. Henry Medina, Silvia Alvarez, Lucy Ruiz y Jeffreey David Ehrenreich. Etnografías mínimas del Ecuador: tsachila, cachis, cholo, cofán, awá-coaiquer. Quito: Abya Yala.

Ventura, Monserrath. 2012: "Chamanismo, Liderazgo y poder indígena, el Caso de la Nacionalidad Tsáchila». Revista Española de Antropología Americana, 42, 1: 91-106.

Yrigoyen, Raquel. 1999. Pautas de coordinación entre el derecho indígena y el derecho estatal. Guatemala: Fundación Myrna Mack.

Rappaport, Joanne. 2012. "Así lo parece por su aspecto. Fisiognomía y construcción de la diferencia en la Bogotá colonial». Acceso el 13 de marzo: http://www.scielo.org.co/scielo.php?script=sci_arttext\&pid=S179424892012000200002 


\section{Copyright}

Deusto Journal of Human Rights / Revista Deusto de Derechos Humanos is an Open Access journal; which means that it is free for full and immediate access, reading, search, download, distribution, and reuse in any medium only for non-commercial purposes and in accordance with any applicable copyright legislation, without prior permission from the copyright holder (University of Deusto) or the author; provided the original work and publication source are properly cited (Issue number, year, pages and DOI if applicable) and any changes to the original are clearly indicated. Any other use of its content in any medium or format, now known or developed in the future, requires prior written permission of the copyright holder.

\section{Derechos de autoría}

Deusto Journal of Human Rights / Revista Deusto de Derechos Humanos es una revista de Acceso Abierto; lo que significa que es de libre acceso en su integridad inmediatamente después de la publicación de cada número. Se permite su lectura, la búsqueda, descarga, distribución y reutilización en cualquier tipo de soporte sólo para fines no comerciales y según lo previsto por la ley; sin la previa autorización de la Editorial (Universidad de Deusto) o la persona autora, siempre que la obra original sea debidamente citada (número, año, páginas y DOI si procede) y cualquier cambio en el original esté claramente indicado. Cualquier otro uso de su contenido en cualquier medio o formato, ahora conocido o desarrollado en el futuro, requiere el permiso previo por escrito de la persona titular de los derechos de autoría. 